\title{
A Survey of Psychological Responses During the Coronavirus Disease 2019 (COVID-19) Epidemic among Chinese Police Officers in Wuhu
}

This article was published in the following Dove Press journal: Risk Management and Healthcare Policy

\author{
Lili Yuan (D) \\ Lele $\mathrm{Zhu}^{2}$ \\ Fangfang Chen ${ }^{3}$ \\ Qian Cheng ${ }^{2}$ \\ Qian Yang' \\ Zhiming Zhou Zhou' \\ Yujuan Zhu' \\ Yigao $\mathrm{Wu}^{4}$ \\ Yong Zhou ${ }^{2}$ \\ Xiaojuan $\mathrm{Zha}^{2}$ \\ 'Department of Neurology, First \\ Affiliated Hospital of Wannan Medical \\ College, Wuhu, Anhui Province, People's \\ Republic of China; ${ }^{2}$ Health Management \\ Center, First Affiliated Hospital of \\ Wannan Medical College, Wuhu, Anhui \\ Province, People's Republic of China; \\ ${ }^{3}$ Department of Psychology, Fourth \\ People's Hospital of Wuhu, Wuhu, Anhui \\ Province, People's Republic of China; \\ ${ }^{4}$ Department of Psychology, First \\ Affiliated Hospital of Wannan Medical \\ College, Wuhu, Anhui Province, People's \\ Republic of China
}

Background: The outbreak of coronavirus disease 2019 (COVID-19) has presented serious threats to people's health and lives. Police officers are bravely fighting on the front lines of the epidemic. The main purpose of this study was to assess the prevalence and severity of psychological responses among police officers during the COVID-19 pandemic and find influencing factors in depression and anxiety.

Methods: A cross-sectional online questionnaire was administered to police officers in Wuhu through WeChat, and data were collected between March 10 and 26, 2020. A total of 3,561 questionnaires were received, of which 3,517 were considered valid. The questionnaires included demographic information and a psychological survey. The depression scale of the Patient Health QuestionnaireQ9) and Generalized Anxiety Disorder scale were utilized to assess depression and anxiety, respectively.

Results: The mean depression score of participants was $4.10 \pm 4.87$ (0-27), and 12.17\%had moderate-severe depression. The mean anxiety score of participants was $3.59 \pm 4.228(0-21)$, and $8.79 \%$ had moderate-severe anxiety. Older and married police officers were at higher risk of anxiety. Those with a bachelor's degree or above, living near the city center, and taking sleeping pills were at greater risk of depression and anxiety. Auxiliary police had lower depression and anxiety scores. Depression scores were strongly correlated withanxiety scores $(r=0.863, p<0.001)$.

Conclusion: Our findings identify factors associated with higher levels of depression and anxiety that can be utilized to develop psychological interventions to improve the mental health of vulnerable populations during the COVID-19 pandemic.

Keywords: COVID-19, police officers, psychological responses, anxiety, depression

\section{Background}

Coronavirus disease 2019 (COVID-19) has been declared a global pandemic. ${ }^{1}$ In China, COVID-19 was first identified in Wuhan in December 2019, and had spread to all 34 regions of China by 30 January 2020. The outbreak of COVID-19 has caused serious threats to people's health and lives. Therefore, the Chinese government has imposed unprecedented strict quarantine measures that have put large numbers of people in isolation and disrupted their way of life. This public-health emergency has put people's physical and mental health under unprecedented threat. $^{2,3}$ A recent study of the Chinese general population demonstrated that $53.8 \%$ of respondents rated the psychological impact of COVID-19 as moderate or severe, $16.5 \%$ reported moderate-severe depressive symptoms, and $28.8 \%$
Correspondence: Lele Zhu; Xiaojuan Zha Email 18297301582@I63.com;

I24026094I@qq.com
Risk Management and Healthcare Policy 2020:I3 2689-2697 
reported moderate-severe anxiety symptoms during the initial stage of the epidemic., ${ }^{2,4}$

The increasing numbers of patients and suspected cases have caused public worry about becoming infected. ${ }^{5}$ There have been a lot of studies focused on the psychological state of health-care workers. ${ }^{6-9}$ In addition to health-care workers, police officers are equally bravely fighting on the front lines of the epidemic. ${ }^{10,11}$ Unfortunately, little attention has been paid to the psychological responses of police officers. Being a police officer is one of the most mentally taxing jobs, requiring long-time and frequent shifts, the threat of violence, heightened vigilance, and a lack of public support. ${ }^{12}$ As first responders to emergencies, police officers not only face a higher risk of infection than the general public but also suffer from fatigue caused by working overtime and the pressures of responsibility. The sudden disruption of society resulting from the COVID-19 pandemic may impact the mental health of police officers and lead to a wide variety of psychological problems, such as anxiety and depression.

This is the first survey of psychological response in police officers of China during the tumultuous time of the COVID-19 outbreaks. A self-report questionnaire in online form was used to investigate anxiety and depression among police officers including criminal police, security police, special police, traffic police, command center and logistics support staff, those engaged in organizational, internal, and political security, prison guards, and auxiliary police in Wuhu between March 10 and 26, 2020. The main purpose of the study was to measure the prevalence and severity of psychological responses among police officers during the COVID-19 epidemic and find influencing factors in depression and anxiety. The survey helped to offer a concrete basis for implementing pertinent mental health-intervention measures to deal with the challenge effectively.

\section{Methods}

\section{Study Design and Participants}

A cross-sectional online questionnaire was administered to all police officers involved in epidemic prevention and control from five districts (Yijiang, Sanshan, Jiujiang, Jinghu, and Jingkai) and four counties (Wuhu, Nanling, Fanchang, and Wuwei) in Wuhu through WeChat, and data collection began on March 10, after the WHO had announced the threat of COVID-19 becoming a pandemic may be a reality on 9 March 2020. Data were collected from 10 March to 26 March. All participants were told that the information they would provide would not be disclosed. When an officer had completed the self-reported questionnaire, psychological status results would be displayed automatically on their mobile phone. Our institution would provide psychological intervention services to those who needed it. These participants voluntarily took part in the survey with online informed consent, which was approved by the ethics committee of the First Affiliated Hospital of Wannan Medical College. Inclusion criteria were police officers, including criminal, security, regimental, traffic, command center and logistics support, organizational, internal, and political security, prison guards, and auxiliary police in Wuhu participating in COVID-19 prevention and control, between 18 and 60 years old, understanding the questionnaire, and no diagnosed neuropsychiatric disorders. A total of 3,561 questionnaires were received in the study, of which 3,517 were considered valid (98.76\%).

\section{Questionnaires}

\section{Demographic Characteristics}

The demographic data were collected by officers providing their personal information online. This information comprised age, sex, education, marital status, current location, position, telephone number, and use of sleeping pills in the last month.

\section{Psychological Surveys Depression Survey}

The nine-item Patient Health Questionnaire (PHQ9), which comprises 9 items with scores between 0 and 27, is a widely used self-report tool for measuring depression during the previous 2 weeks. Participants completed the question using a four-point rating scale from 0 (not at all) to 3 (every day) through WeChat according to their own psychological status. The total score was subdivided into five categories: minimal $(0-4)$, mild (5-9), moderate (10-14), moderate-severe (1519), and severe (20-27). Cronbach's $\alpha$-coefficient for the Chinese version of the PHQ9 was 0.85 . Using 10 as a cutoff, sensitivity and specificity of the GAD7 were $93.33 \%$ and $96.83 \%$, respectively. ${ }^{13}$

\section{Anxiety Survey}

The seven-item Generalized Anxiety Disorder (GAD7) scale, which comprises seven items with scores between 0 and 21, has proven to be an effective tool for assessing anxiety in the last 2 weeks. Participants responded to questions using a four-point rating scale from 0 (not at all) to 3 (every day) through WeChat according to their own psychological status. The total score was divided into four 
categories: minimal (0-4), mild (5-9), moderate (10-14), and severe (15-21). Cronbach's $\alpha$ - coefficient for GAD7 was 0.898 , and test-retest reliability was 0.856 . Using 10 as a cutoff, sensitivity and specificity of the GAD7 were $86.2 \%$ and $95.5 \%$, respectively $(\alpha=0.825){ }^{14}$

\section{Statistical Analysis}

Statistical analysis was carried out using SPSS version 22.0. Quantitative data are expressed as means \pm SD and qualitative data as percentages. One-way ANOVA and rank-sum tests were used in univariate analysis. Risk factors were analyzed using ordered multivariate logistic regression and ORs and 95\% CIs calculated. Scheff's comparison was used to analyze age differences between groups with different degrees of depression and between groups with different degrees of anxiety. All statistical tests were two-sided, with $\alpha=0.05$.

\section{Results}

\section{Description of Sample}

Demographic data of the 3,517 officers recruited are shown in Table 1. There were 557 females $(15.8 \%)$ and 2,960 males $(84.2 \%)$. The average age was $36.20 \pm 9.32$ years, and education of bachelor's or above accounted for the largest proportion (44.1\%), indicating that those investigated were mainly middle-aged and had a good education. Most got married (78.7\%), and their families were stable. Based on location and police classification, Jinghu district (18.3\%) and security police (26.8\%) accounted for the highest proportions, respectively.

\section{Prevalence of Depression and Influencing Factors in Police Officers}

Survey data from the PHQ9 are shown in Table 2. The mean PHQ9 score of the participants was $4.10 \pm 4.87$ (0-27), and $12.17 \%$ had moderate-severe depression. In Table 2, we can see the distribution of the degree of depression (PHQ9 scores). Age, education, marital status, location, police classification, and use of sleeping pills were significantly associated with the degree of depression $(p=0.001, p<0.001$, $p=0.002, p<0.001, p<0.001$, and $p<0.001$, respectively). There was a significant difference between ages in the minimal-depression group and the mild-depression group $(p=0.002)$, but we did not find a significant difference between ages in the minimal-depression group and the other depression groups (moderate, moderate-severe, and severe). Ordered multivariate logistic regression results
Table I Demographic characteristic of participants

\begin{tabular}{|c|c|c|c|}
\hline & Demographics & $\mathbf{n}$ & $\begin{array}{l}\text { Percentage } \\
\text { (\%) }\end{array}$ \\
\hline Sex & $\begin{array}{l}\text { Female } \\
\text { Male }\end{array}$ & $\begin{array}{l}557 \\
2,960\end{array}$ & $\begin{array}{l}15.8 \\
84.2\end{array}$ \\
\hline Education & $\begin{array}{l}\text { Senior high school or below } \\
\text { Academy } \\
\text { Bachelor's or above }\end{array}$ & $\begin{array}{l}760 \\
1,207 \\
1,550\end{array}$ & $\begin{array}{l}21.6 \\
34.3 \\
44.1\end{array}$ \\
\hline $\begin{array}{l}\text { Marital } \\
\text { status }\end{array}$ & $\begin{array}{l}\text { Married } \\
\text { Unmarried } \\
\text { Others }\end{array}$ & $\begin{array}{l}2,769 \\
692 \\
56\end{array}$ & $\begin{array}{l}78.7 \\
19.7 \\
1.6\end{array}$ \\
\hline Location & $\begin{array}{l}\text { Yijiang district } \\
\text { Wuhu county } \\
\text { Sanshan district } \\
\text { Nanling county } \\
\text { Jiujiang district } \\
\text { Jinghu district } \\
\text { Jingkai district } \\
\text { Fanchang county } \\
\text { Wuwei county }\end{array}$ & $\begin{array}{l}334 \\
467 \\
169 \\
387 \\
460 \\
645 \\
97 \\
360 \\
598\end{array}$ & $\begin{array}{l}9.5 \\
13.3 \\
4.8 \\
11 \\
13.1 \\
18.3 \\
2.8 \\
10.2 \\
17\end{array}$ \\
\hline $\begin{array}{l}\text { Police } \\
\text { classification }\end{array}$ & $\begin{array}{l}\text { Criminal } \\
\text { Security } \\
\text { Regimental } \\
\text { Traffic } \\
\text { Command center and } \\
\text { logistics support } \\
\text { Organizational, internal, and } \\
\text { political security } \\
\text { Prison guards } \\
\text { Auxiliary }\end{array}$ & $\begin{array}{l}330 \\
942 \\
341 \\
727 \\
205 \\
198 \\
93 \\
681\end{array}$ & $\begin{array}{l}9.4 \\
26.8 \\
9.7 \\
20.7 \\
5.8 \\
\\
5.6 \\
2.6 \\
19.4\end{array}$ \\
\hline $\begin{array}{l}\text { Use of } \\
\text { sleeping pills }\end{array}$ & $\begin{array}{l}\text { No } \\
\text { Yes }\end{array}$ & $\begin{array}{l}3,401 \\
116\end{array}$ & $\begin{array}{l}96.7 \\
3.3\end{array}$ \\
\hline
\end{tabular}

revealed that those with senior high school or below or academy education had lower PHQ9 scores than those with bachelor's degrees or above (respectively, OR 0.260, $p<0.001$; OR 0.481, $p<0.001$ ). Officers in Yijiang and Jinghu districts had higher PHQ9 scores than those in Wuwei county (respectively, OR 1.426, $p=0.013$; OR $1.354, p=0.013$ ). Officers in Wuhu county had lower PHQ9 scores than those in Wuwei county (OR 0.664, $p=0.004$ ). Criminal police, security police, those engaged in organizational, internal, and political security, and prison guards had higher PHQ9 scores than auxiliary police (respectively, OR $1.581, p=0.001$; OR 1.887, $p<0.001$; OR 1.401, $p=0.047$; OR $1.704, p=0.017$ ). Participants who did not take sleeping pills had lower PHQ9 scores than those who did (OR 0.150, $p<0.001$; Table 3). 
Table 2 Univariate analysis results of the PHQ7

\begin{tabular}{|c|c|c|c|c|c|c|c|}
\hline & & $\begin{array}{l}\text { Minimal } \\
(0-4)\end{array}$ & Mild (5-9) & $\begin{array}{l}\text { Moderate } \\
(10-14)\end{array}$ & $\begin{array}{l}\text { Moderate- } \\
\text { Severe (15-19) }\end{array}$ & $\begin{array}{l}\text { Severe } \\
(20-27)\end{array}$ & $\mathbf{p}$ \\
\hline Age, years & & $35.60 \pm 9.61$ & $37.07 \pm 8.79$ & $36.78 \pm 8.38$ & $37.5 I \pm 8.92$ & $38.12 \pm 8.76$ & 0.001 \\
\hline Sex & $\begin{array}{l}\text { Female } \\
\text { Male }\end{array}$ & $\begin{array}{l}339(60.9 \%) \\
1,906(64.4 \%)\end{array}$ & $\begin{array}{l}145(26.0 \%) \\
699(23.6 \%)\end{array}$ & $\begin{array}{l}50(9.0 \%) \\
216(7.3 \%)\end{array}$ & $\begin{array}{l}15(2.7 \%) \\
95(3.2 \%)\end{array}$ & $\begin{array}{l}8(1.4 \%) \\
44(1.5 \%)\end{array}$ & 0.378 \\
\hline Education & $\begin{array}{l}\text { Senior high school or below } \\
\text { Academy } \\
\text { Bachelor's or above }\end{array}$ & $\begin{array}{l}617(81.2 \%) \\
859(71.2 \%) \\
769(49.6 \%)\end{array}$ & $\begin{array}{l}102(13.4 \%) \\
233(19.3 \%) \\
509(32.8 \%)\end{array}$ & $\begin{array}{l}27(3.6 \%) \\
63(5.2 \%) \\
176(66.2 \%)\end{array}$ & $\begin{array}{l}12(1.6 \%) \\
36(3.0 \%) \\
62(4.0 \%)\end{array}$ & $\begin{array}{l}2(0.3 \%) \\
16(1.3 \%) \\
34(2.2 \%)\end{array}$ & $<0.001$ \\
\hline $\begin{array}{l}\text { Marital } \\
\text { status }\end{array}$ & $\begin{array}{l}\text { Married } \\
\text { Unmarried } \\
\text { Others }\end{array}$ & $\begin{array}{l}\mathrm{I}, 7 \mathrm{I} 5(6 \mathrm{I} .9 \%) \\
494(7 \mathrm{I} .4 \%) \\
36(64.3 \%)\end{array}$ & $\begin{array}{l}700(25.3 \%) \\
133(19.2 \%) \\
11(19.6 \%)\end{array}$ & $\begin{array}{l}216(7.8 \%) \\
45(6.5 \%) \\
5(8.9 \%)\end{array}$ & $\begin{array}{l}92(3.3 \%) \\
15(2.2 \%) \\
3(5.4 \%)\end{array}$ & $\begin{array}{l}46(1.7 \%) \\
5(0.7 \%) \\
I(1.8 \%)\end{array}$ & 0.002 \\
\hline Location & $\begin{array}{l}\text { Yijiang district } \\
\text { Wuhu county } \\
\text { Sanshan district } \\
\text { Nanling county } \\
\text { Jiujiang district } \\
\text { Jinghu district } \\
\text { Jingkai district } \\
\text { Fanchang county } \\
\text { Wuwei county }\end{array}$ & $\begin{array}{l}177(53.0 \%) \\
349(74.7 \%) \\
115(68.0 \%) \\
250(64.6 \%) \\
292(63.5 \%) \\
372(57.7 \%) \\
54(55.7 \%) \\
224(62.2 \%) \\
412(68.9 \%)\end{array}$ & $\begin{array}{l}112(33.5 \%) \\
84(18.0 \%) \\
35(20.7 \%) \\
87(22.5 \%) \\
117(25.4 \%) \\
168(26.0 \%) \\
30(30.9 \%) \\
83(23.1 \%) \\
128(21.4 \%)\end{array}$ & $\begin{array}{l}30(9.0 \%) \\
19(4.1 \%) \\
12(7.1 \%) \\
35(9.0 \%) \\
31(6.7 \%) \\
59(9.1 \%) \\
10(10.3 \%) \\
30(8.3 \%) \\
40(6.7 \%)\end{array}$ & $\begin{array}{l}9(2.7 \%) \\
9(1.9 \%) \\
5(3.0 \%) \\
10(2.6 \%) \\
15(3.3 \%) \\
32(5.0 \%) \\
1(1.0 \%) \\
16(4.4 \%) \\
13(2.2 \%)\end{array}$ & $\begin{array}{l}6(1.8 \%) \\
6(1.3 \%) \\
2(1.2 \%) \\
5(1.3 \%) \\
5(1.1 \%) \\
14(2.2 \%) \\
2(2.1 \%) \\
7(1.9 \%) \\
5(0.8 \%)\end{array}$ & $<0.001$ \\
\hline $\begin{array}{l}\text { Police } \\
\text { classification }\end{array}$ & $\begin{array}{l}\text { Criminal } \\
\text { Security } \\
\text { Regimental } \\
\text { Traffic } \\
\text { Command center and logistics } \\
\text { support } \\
\text { Organizational, internal, and } \\
\text { political security } \\
\text { Prison guards } \\
\text { Auxiliary }\end{array}$ & $\begin{array}{l}164(49.7 \%) \\
53 \mid(56.4 \%) \\
229(67.2 \%) \\
537(73.9 \%) \\
143(69.8 \%) \\
107(54.0 \%) \\
46(49.5 \%) \\
488(71.7 \%)\end{array}$ & $\begin{array}{l}106(37.6 \%) \\
250(26.5 \%) \\
85(24.9 \%) \\
127(17.5 \%) \\
48(23.4 \%) \\
71(35.9 \%) \\
25(26.9 \%) \\
132(19.4 \%)\end{array}$ & $\begin{array}{l}46(13.9 \%) \\
94(10.0 \%) \\
21(6.2 \%) \\
34(4.7 \%) \\
8(3.9 \%) \\
15(7.6 \%) \\
15(16.1 \%) \\
33(4.8 \%)\end{array}$ & $\begin{array}{l}13(3.9 \%) \\
42(4.5 \%) \\
5(1.5 \%) \\
23(3.2 \%) \\
4(2.0 \%) \\
3(1.5 \%) \\
4(4.3 \%) \\
16(2.3 \%)\end{array}$ & $\begin{array}{l}\text { I }(0.3 \%) \\
25(2.7 \%) \\
\text { I }(0.3 \%) \\
6(0.8 \%) \\
2(1.0 \%) \\
2(1.0 \%) \\
3(3.2 \%) \\
12(1.8 \%)\end{array}$ & $<0.001$ \\
\hline $\begin{array}{l}\text { Use of } \\
\text { sleeping pills }\end{array}$ & $\begin{array}{l}\text { No } \\
\text { Yes }\end{array}$ & $\begin{array}{l}2,222(65.3 \%) \\
23(19.8 \%)\end{array}$ & $\begin{array}{l}807(23.7 \%) \\
37(31.9 \%)\end{array}$ & $\begin{array}{l}237(7.0 \%) \\
29(25.0 \%)\end{array}$ & $\begin{array}{l}100(2.9 \%) \\
10(8.6 \%)\end{array}$ & $\begin{array}{l}35(1.0 \%) \\
17(14.7 \%)\end{array}$ & $<0.001$ \\
\hline
\end{tabular}

Prevalence of Anxiety and Influencing Factors in Police Officers

Survey data from the GAD7 are shown in Table 4. The mean GAD7 score of the participants was 3.59 \pm 4.228 (0-21), and $8.79 \%$ had moderate-severe anxiety. In Table 4, we can see the distribution of anxiety (GAD7 score) of the officers. Age, sex, education, marital status, location, police classification, and use of sleeping pills were significantly associated with degree of anxiety $(p<0.001, p=0.018, p<0.001, \mathrm{p}<0.001, p<0.001, p<0.001$, and $p<0.001$, respectively). There were significant differences between age in the minimal-anxiety group 
Table 3 Ordered multivariate logistic regression for depression

\begin{tabular}{|c|c|c|c|c|c|c|c|c|}
\hline & & $\beta$ & SE & Wald & $p$ & OR & \multicolumn{2}{|c|}{$95 \% \mathrm{Cl}$} \\
\hline Age & & 0.009 & 0.005 & 3.483 & 0.062 & 1.009 & 1.000 & 1.018 \\
\hline Sex & Female & 0.009 & 0.102 & 0.007 & 0.931 & 1.009 & 0.826 & 1.231 \\
\hline \multirow[t]{2}{*}{ Education } & Senior high school or below & -1.347 & 0.110 & $|48.72|$ & $<0.001$ & 0.260 & 0.210 & 0.323 \\
\hline & Academy & -0.732 & 0.083 & 77.264 & $<0.001$ & $0.48 \mathrm{I}$ & 0.409 & 0.566 \\
\hline \multirow[t]{3}{*}{ Marital status } & & & & & & & & \\
\hline & Unmarried & -0.27 & 0.289 & 0.875 & 0.35 & 0.763 & 0.433 & 1.344 \\
\hline & Others & -0.184 & 0.108 & 2.824 & 0.087 & 0.832 & 0.673 & 1.027 \\
\hline \multirow[t]{9}{*}{ Location } & & & & & & & & \\
\hline & Yijiang district & 0.355 & 0.144 & 6.104 & 0.013 & 1.426 & 1.076 & 1.889 \\
\hline & Wuhu county & -0.410 & 0.143 & 8.166 & 0.004 & 0.664 & 0.501 & 0.879 \\
\hline & Sanshan district & -0.041 & 0.192 & 0.046 & 0.830 & 0.960 & 0.658 & 1.398 \\
\hline & Nanling county & 0.108 & 0.142 & 0.578 & 0.447 & 1.114 & 0.843 & 1.473 \\
\hline & Jiujiang district & 0.122 & 0.136 & 0.801 & 0.371 & 1.130 & 0.865 & 1.476 \\
\hline & Jinghu district & 0.303 & 0.122 & 6.127 & 0.013 & 1.354 & 1.065 & 1.719 \\
\hline & Jingkai district & 0.303 & 0.227 & 1.777 & 0.183 & 1.354 & 0.868 & 2.111 \\
\hline & Fanchang county & 0.085 & 0.144 & 0.350 & 0.554 & 1.089 & 0.821 & 1.445 \\
\hline \multirow[t]{8}{*}{ Police classification } & & & & & & & & \\
\hline & Criminal & 0.458 & 0.142 & 10.447 & 0.001 & 1.581 & 1.197 & 2.088 \\
\hline & Security & 0.635 & 0.111 & 32.546 & $<0.001$ & 1.887 & 1.517 & 2.347 \\
\hline & Regimental & 0.069 & 0.154 & 0.200 & 0.655 & 1.071 & 0.792 & 1.448 \\
\hline & Traffic & -0.111 & 0.125 & 0.799 & 0.371 & 0.895 & 0.700 & 1.142 \\
\hline & Command center and logistics support & -0.116 & 0.183 & 0.404 & 0.525 & 0.890 & 0.623 & 1.274 \\
\hline & Organizational, internal, and political security & 0.337 & 0.170 & 3.938 & 0.047 & 1.401 & 1.004 & 1.954 \\
\hline & Prison guards & 0.533 & 0.224 & 5.681 & 0.017 & 1.704 & 1.100 & 2.643 \\
\hline \multirow[t]{2}{*}{ Use of sleeping pills } & & & & & & & & \\
\hline & No & -1.899 & 0.176 & 116.408 & $<0.001$ & 0.150 & 0.106 & 0.211 \\
\hline
\end{tabular}

and the other three groups (mild, moderate, and severe: $p=0.001, p=0.001$, and $p=0.006$, respectively), but there was no significant difference among ages in the mild, moderate, and severe groups. Ordered multivariate logistic regression results revealed that the risk of higher anxiety scores increased with age (OR 1.010, $p=0.044)$. Those who had had senior high school or below or academy education had lower GAD7 scores than those with bachelor's degrees or above (OR 0.194, $p<0.001$ and OR 0.407, $p<0.001$, respectively). Unmarried officers had lower GAD7 score than married ones (OR 0.614, $p<0.001$ ). Those in Wuhu county had lower GAD7 scores than those in Wuwei county (OR 0.605, $p=0.001$ ). Security police and prison guards had higher GAD7 scores than auxiliary police, (OR 1.822, $p<0.001$ and OR 1.634, $p=0.032$, respectively). Participants who did not take sleeping pills had lower GAD7 scores than those who did (OR 0.186, $p<0.001$; Table 5).

\section{Relationship Between PHQ9 and GAD7}

\section{Scores}

PHQ9 scores were strongly correlated with GAD7 scores $(r=0.863, p<0.001$; Figure 1$)$.

\section{Discussion}

COVID-19 is now a worldwide pandemic. Police officers are participating in a war against the epidemic, which increases their likelihood of contracting COVID-19 and aggravates their mental problems. ${ }^{11,12,15}$ Our study found $12.17 \%$ of them had moderate-severe depression and $8.79 \%$ had moderate-severe anxiety in Wuhu city. Depression symptoms are related to anxiety symptoms. Tan et al found that about $3.7 \%$ and $3.8 \%$ of respondents reported moderate-severe depression and anxiety, respectively, in the workforce that returned to work after lockdown and quarantine in Chongqing during the pandemic, including workers, technical staff, executives, sales and 
Table 4 Univariate analysis results of the GAD7

\begin{tabular}{|c|c|c|c|c|c|c|}
\hline & & $\begin{array}{l}\text { Minimal } \\
(0-4)\end{array}$ & $\begin{array}{l}\text { Mild } \\
(5-9)\end{array}$ & $\begin{array}{l}\text { Moderate } \\
(10-14)\end{array}$ & $\begin{array}{l}\text { Severe } \\
(20-27)\end{array}$ & $\mathbf{p}$ \\
\hline Age, years & & $35.60 \pm 9.66$ & $37.01 \pm 8.49$ & $38.09 \pm 8.52$ & $\begin{array}{l}38.88 \\
\pm 8.36\end{array}$ & $<0.001$ \\
\hline Sex & $\begin{array}{l}\text { Female } \\
\text { Male }\end{array}$ & $\begin{array}{l}342 \text { (6I.4\%) } \\
\text { I,97। (66.6\%) }\end{array}$ & $\begin{array}{l}165(29.6 \%) \\
730(24.7 \%)\end{array}$ & $\begin{array}{l}4 \mid(7.4 \%) \\
\mid 78(6.0 \%)\end{array}$ & $\begin{array}{l}9(1.6 \%) \\
81(2.7 \%)\end{array}$ & 0.018 \\
\hline Education & $\begin{array}{l}\text { Senior high school or below } \\
\text { Academy } \\
\text { Bachelor's or above }\end{array}$ & $\begin{array}{l}643(84.6 \%) \\
890(73.7 \%) \\
780(50.3 \%)\end{array}$ & $\begin{array}{l}90(11.8 \%) \\
242(20.0 \%) \\
563(36.3 \%)\end{array}$ & $\begin{array}{l}21(2.8 \%) \\
51(4.2 \%) \\
147(9.5 \%)\end{array}$ & $\begin{array}{l}6(0.8 \%) \\
24(2.0 \%) \\
60(3.9 \%)\end{array}$ & $<0.001$ \\
\hline Marital status & $\begin{array}{l}\text { Married } \\
\text { Unmarried } \\
\text { Others }\end{array}$ & $\begin{array}{l}I, 74 \mathrm{I}(62.8 \%) \\
536(77.5 \%) \\
37(66.1 \%)\end{array}$ & $\begin{array}{l}755(27.2 \%) \\
129(18.6 \%) \\
12(21.4 \%)\end{array}$ & $\begin{array}{l}192(6.9 \%) \\
22(3.2 \%) \\
5(8.9 \%)\end{array}$ & $\begin{array}{l}83(3.0 \%) \\
5(0.7 \%) \\
2(3.6 \%)\end{array}$ & $<0.001$ \\
\hline Location & $\begin{array}{l}\text { Yijiang district } \\
\text { Wuhu county } \\
\text { Sanshan district } \\
\text { Nanling county } \\
\text { Jiujiang district } \\
\text { Jinghu district } \\
\text { Jingkai district } \\
\text { Fanchang county } \\
\text { Wuwei county }\end{array}$ & $\begin{array}{l}189(56.6 \%) \\
359(76.9 \%) \\
120(71.0 \%) \\
260(67.2 \%) \\
314(68.3 \%) \\
375(58.1 \%) \\
53(54.6 \%) \\
227(63.1 \%) \\
416(69.6 \%)\end{array}$ & $\begin{array}{l}106(31.7 \%) \\
83(17.8 \%) \\
33(19.5 \%) \\
89(23.0 \%) \\
118(25.7 \%) \\
194(30.1 \%) \\
34(35.1 \%) \\
96(26.7 \%) \\
142(23.7 \%)\end{array}$ & $\begin{array}{l}29(8.7 \%) \\
15(3.2 \%) \\
11(6.5 \%) \\
30(7.8 \%) \\
20(4.3 \%) \\
58(9.0 \%) \\
6(6.2 \%) \\
25(6.9 \%) \\
25(4.2 \%)\end{array}$ & $\begin{array}{l}10(3.0 \%) \\
10(2.1 \%) \\
5(3.0 \%) \\
8(2.1 \%) \\
8(1.7 \%) \\
18(2.8 \%) \\
4(2.5 \%) \\
12(3.3 \%) \\
15(2.5 \%)\end{array}$ & $<0.001$ \\
\hline Police classification & $\begin{array}{l}\text { Criminal } \\
\text { Security } \\
\text { Regimental } \\
\text { Traffic } \\
\text { Command center and logistics support } \\
\text { Organizational, internal, and political security } \\
\text { Prison guards } \\
\text { Auxiliary police }\end{array}$ & $\begin{array}{l}174(52.7 \%) \\
552(58.6 \%) \\
249(73.0 \%) \\
533(73.3 \%) \\
153(74.6 \%) \\
108(54.5 \%) \\
48(51.6 \%) \\
496(72.8 \%)\end{array}$ & $\begin{array}{l}\text { I24 (37.6\%) } \\
226(28.2 \%) \\
79(23.3 \%) \\
146(20.1 \%) \\
4 I(20.0 \%) \\
73(36.9 \%) \\
27(29.0 \%) \\
139(20.4 \%)\end{array}$ & $\begin{array}{l}28(8.5 \%) \\
79(8.4 \%) \\
8(2.3 \%) \\
34(4.7 \%) \\
8(3.9 \%) \\
13(6.6 \%) \\
14(15.5 \%) \\
35(5.1 \%)\end{array}$ & $\begin{array}{l}4(1.2 \%) \\
45(4.8 \%) \\
5(1.5 \%) \\
14(1.9 \%) \\
3(1.5 \%) \\
4(2.0 \%) \\
4(4.3 \%) \\
11(1.6 \%)\end{array}$ & $<0.001$ \\
\hline Use of sleeping pills & $\begin{array}{l}\text { No } \\
\text { Yes }\end{array}$ & $\begin{array}{l}2,287(67.2 \%) \\
26(22.4 \%)\end{array}$ & $\begin{array}{l}845(24.9 \%) \\
50(43.1 \%)\end{array}$ & $\begin{array}{l}197(5.8 \%) \\
22(19.0 \%)\end{array}$ & $\begin{array}{l}72(2.1 \%) \\
18(15.5 \%)\end{array}$ & $<0.001$ \\
\hline
\end{tabular}

marking, management, and others. ${ }^{16}$ The reason that the prevalence of rates of anxiety and depression in police officers are higher than that in those industries may be that police officers face greater work pressure and risk of exposure to COVID-19. Another study found that $16.5 \%$ of respondents reported moderate-severe depressive symptoms and $28.8 \%$ reported moderate-severe anxiety symptoms during the COVID-19 pandemic in the Chinese general population. ${ }^{4}$ Some of the population were in high-risk areas, and the authors did not compare anxiety and depression between high-risk and low-risk areas. We hypothesized that our sample came from lowrisk areas, so the rates of anxiety and depression were less than in the general Chinese population. Moreover, our investigation was conducted from March 10 to March 28. During this period, the epidemic in China had eased slightly compared to February.

The current study, which focused on psychological responses among police officers in Wuhu during the COVID-19 epidemic, found that education was an influencing 
Table 5 Ordered multivariate logistic regression for anxiety

\begin{tabular}{|c|c|c|c|c|c|c|c|c|}
\hline & & $\beta$ & SE & Wald & $p$ & OR & \multicolumn{2}{|c|}{$95 \% \mathrm{Cl}$} \\
\hline Age & & 0.010 & 0.005 & 4.071 & 0.044 & 1.010 & 1.000 & 1.019 \\
\hline Sex & Female & 0.640 & 0.104 & 0.376 & 0.540 & 1.896 & 0.869 & 1.307 \\
\hline \multirow[t]{2}{*}{ Education } & Senior high school or below & -1.640 & 0.118 & 192.854 & $<0.001$ & 0.194 & 0.154 & 0.244 \\
\hline & Academy & -0.900 & 0.860 & 109.763 & $<0.001$ & 0.407 & 0.343 & 0.481 \\
\hline \multirow[t]{3}{*}{ Marital status } & & & & & & & & \\
\hline & Unmarried & -0.294 & 0.295 & 0.993 & 0.319 & 0.745 & 0.419 & 1.342 \\
\hline & Others & -0.487 & 0.115 & $|7.84|$ & $<0.001$ & 0.614 & 0.490 & 0.770 \\
\hline \multirow[t]{9}{*}{ Location } & & & & & & & & \\
\hline & Yijiang district & 0.289 & 0.147 & 3.856 & 0.050 & 1.335 & 1.001 & 1.782 \\
\hline & Wuhu county & -0.503 & 0.147 & 11.489 & 0.001 & 0.605 & 0.452 & 0.809 \\
\hline & Sanshan district & -0.150 & 0.199 & 0.570 & 0.450 & $0.86 I$ & 0.583 & 1.271 \\
\hline & Nanling county & 0.084 & 0.147 & 0.323 & 0.570 & 1.088 & 0.815 & 1.451 \\
\hline & Jiujiang district & -0.078 & 0.142 & 0.301 & 0.583 & 0.925 & 0.700 & 1.223 \\
\hline & Jinghu district & 0.244 & 0.125 & 0.382 & 0.051 & 1.276 & 0.999 & 1.631 \\
\hline & Jingkai district & 0.408 & 0.299 & 0.317 & 0.750 & 1.504 & 0.960 & 2.356 \\
\hline & Fanchang county & 0.064 & 0.148 & 0.186 & 0.666 & 1.066 & 0.798 & 1.425 \\
\hline \multirow[t]{8}{*}{ Police classification } & & & & & & & & \\
\hline & Criminal & 0.267 & 0.146 & 3.331 & 0.068 & 1.306 & 0.980 & 1.740 \\
\hline & Security & 0.600 & 0.115 & 27.276 & 0.000 & 1.822 & 1.455 & 2.282 \\
\hline & Regimental & -0.105 & 0.163 & 0.417 & 0.519 & 0.900 & 0.653 & 1.240 \\
\hline & Traffic & -0.009 & 0.127 & 0.005 & 0.945 & 0.991 & 0.773 & 1.273 \\
\hline & Command center and logistics support & -0.383 & 0.193 & 3.736 & 0.053 & 0.682 & 0.472 & 1.005 \\
\hline & Organizational, internal, and political security & 0.330 & 0.173 & 3.627 & 0.057 & 1.391 & 0.990 & 1.952 \\
\hline & Prison guards & 0.491 & 0.229 & 4.590 & 0.032 & 1.634 & 1.043 & 2.563 \\
\hline \multirow[t]{2}{*}{ Use of sleeping pills } & & & & & & & & \\
\hline & No & -1.681 & 0.180 & 87.436 & $<0.001$ & 0.186 & 0.135 & 0.265 \\
\hline
\end{tabular}

factor in depression and anxiety, and age and marital status influencing factors in anxiety. Police officers with bachelor's degrees or above were at greater risk of depression and anxiety, but no significant association between education and mood symptoms was found in conscripted frontline nurses in Wuhan. ${ }^{7}$ Wang et al found that among the general public, those with no formal education had a greater likelihood of depression during the epidemic. ${ }^{4}$ All police officers had received formal education, with bachelor's or above accounting for the majority, and the psychological status of the group with bachelor's or above should be given more attention. The average age in the minimal-anxiety group was lower than in the other three groups, and age might aggravate the effect of anxiety. However, Moustafa et al showed that levels of anxiety decrease with age in the general population. ${ }^{17}$ We speculate that the older officers had more family responsibilities and worries. It may also be the reason that the risk of anxiety among married police officers is higher than that of people with other marital status.
Wuhu is the second-largest city in Anhui Province, with a population of about 400 million, located along the middle and lower reaches of the Yangtze River. There are five districts and four counties in Wuhu city: namely Jinghu district, Yijiang district, Jiujiang district, Sanshan district, Jingkai district, Wuwei county, Wuhu county, Nanling county, and Fanchang county. During our investigation, a total of 34 COVID-19 diagnoses were been confirmed in Wuhu city: 14 in Wuwei county, six in Jinghu district, three in Nanling county, three in Yijiang district, two in Wuhu county, two in Jingkai district, one in Jiujiang district, and three for Hubei crew members passing through Wuhu.

We found a significant association between location or police classification and mood symptoms. Before the investigation, we guessed that levels of anxiety and depression in police officers were higher in districts with higher contagion statistics. However, when the emotional states of officers from different districts were compared, 


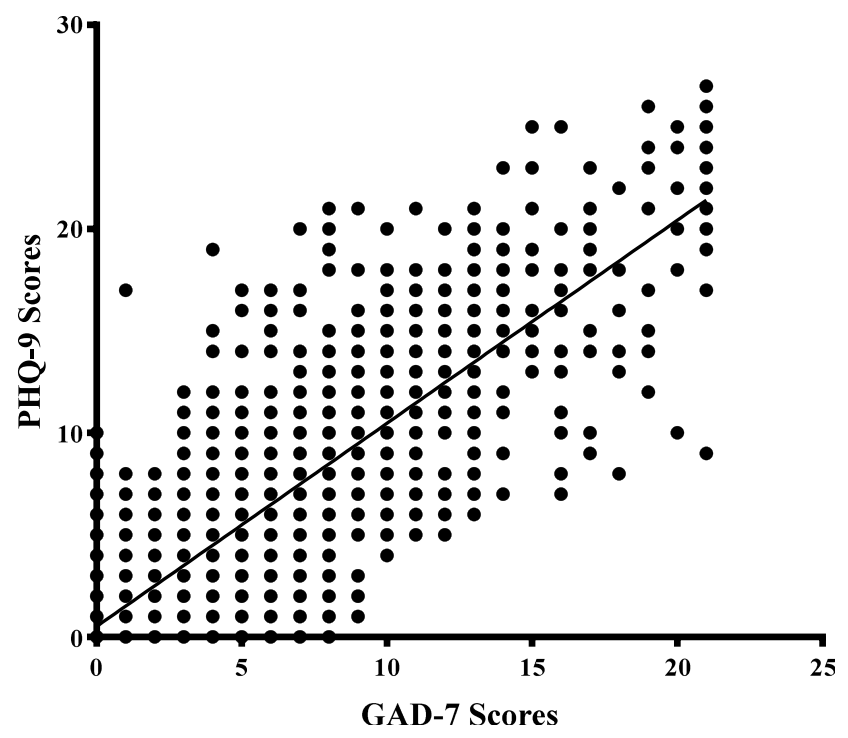

Figure I PHQ9 scores were strongly correlated with GAD7 scores.

the results were not consistent with our hypothesis. Although Wuwei county had the most confirmed cases, the results indicated that depression and anxiety scores of officers in Wuwei county were significantly higher than those in Wuhu County. Police in Yijiang district and Jinghu district had higher depression scores than those in Wuwei county. The reason might be that the locations of the two districts are closer to the city center and the police officers in the two districts have greater work intensity. This result indicated that different working positions had an influence on the psychological state of the officers. Auxiliary police had lower depression and anxiety scores than security police or prison guards, and lower depression scores than the criminal police and those engaged in organizational, internal, and political security. The role of auxiliary police is to assist the daily operation and activities of public security organs, and they have less responsibility and work pressure.

Police officers using sleeping pills were at higher risk of depression and anxiety than those who were not. Taking sleeping pills may indicate that a subject had sleep problems. Poor sleep quality was linked with depression symptoms. ${ }^{7}$ While our study did not analyze the sleep quality of the subjects, previous studies have shown that police officers are exposed to several risk factors, including extended work schedules, shift work, occupational stress, and dangerous events, and thus could develop sleep problems. ${ }^{15}$ The implementation of sleep hygiene-promotion programs in police officers is strongly recommended.
Our survey on the psychological responses of police officers during the COVID-19 epidemic was an investigation during a special period. Inevitably, the current study has several limitations. First of all, it was a cross-sectional study, and we lacked previous measurements of depression and anxiety of the officers. As such, it was hard to determine associations between COVID-19 and depression and anxiety in the study. However, we will continue to follow up on the depression and anxiety of the officers in postpandemic investigation. Second, Wuhu is a low-risk area for COVID-19. At the time of writing, there were 34 confirmed cases and 435 close contacts under medical observation. Although police officers are at risk during epidemic prevention and control, their psychological reactions and stress may still be lower than those in highrisk areas. It is undeniable that the police are making great contributions to society during the pandemic. We call for attention to be paid to psychological responses of police officers during the pandemic and corresponding help and follow-up provided.

\section{Conclusion}

As far as we know, our study is the first survey of psychological responses in police officers during the COVID-19 pandemic. We found that $12.17 \%$ of police officers had moderate-severe depression and $8.79 \%$ moderate-severe anxiety in Wuhu. Police officers with bachelor's degrees or above, located near the city center, and taking sleeping pills were at greater risk of depression and anxiety. Older and married police officers were at higher risk of anxiety, because they had more family responsibilities and worries. The auxiliary police had lower depression and anxiety scores, though they have less responsibility and work pressure. We should identify police officers at high risk of psychological disorders and implement psychological intervention. The findings of this study may provide useful psychological guidance to police officers in other areas of China or outside China dealing with COVID-19.

\section{Abbreviations}

COVID-19, coronavirus disease 2019; GAD7, seven-item Generalized Anxiety Disorder (scale); PHQ9, nine-item Patient Health Questionnaire.

\section{Data-Sharing Statement}

The data used to support the findings of this study are available from the corresponding author upon request. 


\section{Ethical Approval and Consent to Participate}

All participants provided informed consent online. This study was carried out in compliance with the Declaration of Helsinki, and was approved by the ethics committee of the First Affiliated Hospital of Wannan Medical College.

\section{Consent for Publication}

All institutional consent for publication has been provided.

\section{Acknowledgments}

The authors would like to acknowledge and thank the police officers in Wuhu for their participation, service, and assistance in this study.

\section{Funding}

This work was supported by grants from the COVID-19 Epidemic Prevention and Control Special Emergency project of Wuhu City (grant 2020rkxl-2), research project of the First Affiliated Hospital of Wannan Medical College (grant YR202012), and the Scientific Research Foundation of Wannan Medical College(grant WK2019F20). The funding sources had no role in the design, collection, analysis, or interpretation of data or writing the manuscript.

\section{Disclosure}

The authors declare no conflicts of interest.

\section{References}

1. Arden MA, Chilcot J. Health psychology and the coronavirus (COVID-19) global pandemic: A call for research. $\mathrm{Br} J$ Health Psychol. 2020;25(2):231-232. doi:10.1111/bjhp.12414

2. Wang C, Pan R, Wan X, et al. Immediate Psychological Responses and Associated Factors during the Initial Stage of the 2019 Coronavirus Disease (COVID-19) Epidemic among the General Population in China. Int J Environ Res Public Health. 2020;17.

3. Lima CKT, Carvalho PMM, Lima I, et al. The emotional impact of Coronavirus 2019-nCoV (new Coronavirus disease). Psychiatry Res. 2020;287:112915. doi:10.1016/j.psychres.2020.112915
4. Wang C, Pan R, Wan X, et al. A longitudinal study on the mental health of general population during the COVID-19 epidemic in China. Brain Behav Immun. 2020;87:40-48. doi:10.1016/j.bbi.2020.04.028

5. Mazza C, Ricci E, Biondi S, et al. A Nationwide Survey of Psychological Distress among Italian People during the COVID-19 Pandemic: immediate Psychological Responses and Associated Factors. Int J Environ Res Public Health. 2020;17.

6. Wang W, Song W, Xia Z, et al. Sleep Disturbance and Psychological Profiles of Medical Staff and Non-Medical Staff During the Early Outbreak of COVID-19 in Hubei Province, China. Fron Psychiatry. 2020;11:733. doi:10.3389/fpsyt.2020.00733

7. Tu ZH, He JW, Zhou N. Sleep quality and mood symptoms in conscripted frontline nurse in Wuhan, China during COVID-19 outbreak: A cross-sectional study. Medicine. 2020;99:e20769. doi:10. 1097/MD.0000000000020769

8. Zhang WR, Wang K, Yin L, et al. Mental Health and Psychosocial Problems of Medical Health Workers during the COVID-19 Epidemic in China. Psychother Psychosom. 2020;89:242-250. doi:10.1159/000507639

9. Yin Q, Sun Z, Liu T, et al. Posttraumatic stress symptoms of health care workers during the corona virus disease 2019. Clin Psychol Psychother. 2020;27(3):384-395. doi:10.1002/cpp.2477

10. Stanley IH, Hom MA, Joiner TE. A systematic review of suicidal thoughts and behaviors among police officers, firefighters, EMTs, and paramedics. Clin Psychol Rev. 2016;44:25-44. doi:10.1016/j. cpr.2015.12.002

11. Holman G, O'Brien AJ, Thom K. Police and mental health responses to mental health crisis in the Waikato region of New Zealand. Int J Ment Health Nurs. 2018;27(5):1411-1419. doi:10.1111/inm.12440

12. Stogner J, Miller BL, Police Stress MK, Health M. Resiliency during the COVID-19 Pandemic. Am j Criminal Justice. 2020;1-13.

13. Hxzylwzhy SC. Reliability and validity of the patient health questionnaire -9 in Chinese adolescents. Sichuan Mental Health. 2014;27:357-360.

14. XY LC H, Qian J, Cui HS, Wu WY. Reliability and validity of a generalized anxiety disorder scale in general hospital outpatient. Shanghai Arch Psychiatry Res. 2010;22:200-203.

15. Garbarino S, Guglielmi O, Puntoni M, Bragazzi N, Magnavita N. Sleep Quality among Police Officers: implications and Insights from a Systematic Review and Meta-Analysis of the Literature. Int J Environ Res Public Health. 2019;16(5):885. doi:10.3390/ijerph16050885

16. Tan W, Hao F, McIntyre RS, et al. Is returning to work during the COVID-19 pandemic stressful? A study on immediate mental health status and psychoneuroimmunity prevention measures of Chinese workforce. Brain Behav Immun. 2020;87:84-92. doi:10.1016/j. bbi.2020.04.055

17. Moustafa AA, Tindle R, Frydecka D, et al. Impulsivity and its relationship with anxiety, depression and stress. Compr Psychiatry. 2017;74:173-179. doi:10.1016/j.comppsych.2017.01.013
Risk Management and Healthcare Policy is an international, peerreviewed, open access journal focusing on all aspects of public health, policy, and preventative measures to promote good health and improve morbidity and mortality in the population. The journa welcomes submitted papers covering original research, basic science, clinical \& epidemiological studies, reviews and evaluations, guidelines, expert opinion and commentary, case reports and extended reports. The manuscript management system is completely online and includes a very quick and fair peer-review system, which is all easy to use. Visit http://www.dovepress.com/testimonials.php to read real quotes from published authors. 